\title{
Clinical-pathologic factors, as predictor of lymph nodes metastasis in cervical cancer stage IB and IIA
}

\author{
M.Farid Aziz , Andrijono*, Laila Nuranna ${ }^{*}$, Sigit Purbadi ${ }^{*}$, Rukmini Tjipto Mangunkusumo ${ }^{\dagger}$, Budiningsih Siregar ${ }^{\dagger}$, \\ Santoso Cornain ${ }^{\dagger}$, Abdul Bari Saifuddin*, Achmad Tjarta ${ }^{\dagger}$, Bambang Sutrisna $^{\S}$
}

\begin{abstract}
Abstrak
Tujuan penelitian ini adalah untuk mengidentifikasi faktor prediktor metastasis kelenjar getah bening (KGB) pada pasien dengan kanker serviks stadium IB dan IIA. Penelitian dilakukan dari bulan Mei 1996 sampai bulan Desember 2001. Ada 183 pasien kanker serviks dengan stadium menurut FIGO IB dan IIA menjalani operasi histerektomi radikal dan limfadenektomi. Dari pasien tersebut 158 pasien yang dapat dinilai, terdiri dari 43 pasien dengan metastasis KGB dan 115 tanpa metastasis KGB. Rancangan penelitian adalah kasus-kontrol. Kasus adalah pasien dengan metastasis KGB dan kontrol pasien tanpa metastasis KGB. Analisis multivariat dilakukan setelah analisis bivariat. Pada analisis bivariat umur $<39$ tahun, diameter lesi $>4 \mathrm{~cm}$, stadium IIA > $4 \mathrm{~cm}$, histopatologi dengan diferensiasi sedang dan buruk, invasi ke pembuluh darah dan limfa merupakan variabel yang independen terjadinya metastasis KGB dengan nilai $p \leq 0,05$. Tetapi pada analisis multivariat yang muncul sebagai variabel independen adalah umur muda, paritas > 4, diameter lesi, histopatologi adenoskuamosa, dan invasi limfo-vaskular dengan nilai p $\leq 0,05$. Kesimpulan: Usia muda, paritas > 4, stadium IIA > $4 \mathrm{~cm}$, diameter lesi, histopatologi adenoskuamosa, invasi limfa-vaskular merupakan faktor risiko terjadinya metastasis dan dapat dipergunakan sebagai faktor prediktor metastasis KGB. (Med J Indones 2004; 13: 113-8)
\end{abstract}

\begin{abstract}
The aim of this study was to identify possible predictor factors of lymph node metastases in patients with cervical cancer stage IB and IIA. Study was conducted between May 1996 and December 2001. There were 183 patients of cervical cancer with FIGO Stage IB and IIA who were underwent radical hysterectomy and lymphadenectomy. From those 158 patients could be evaluated, consisting 43 patients with node metastases 115 patients without metastases. Research design was case control study. Case was patients with node metastases and control was those without node metastases. Multivariate analysis was made after bivariate analysis. On bivariate analysis age $<39$ years, diameter of lesion $>4 \mathrm{~cm}$, stage IIA $>4 \mathrm{~cm}$, histopathology moderate and poor differentiation, blood and lymphatic vessel invasion were independent variables for node metastases with $p$ value $\leq 0.05$. However, on multivariate analysis younger age, parity $\geq 4$, diameter of lesion, histopathology adenosquamous, and lymph vascular invasion (+) as independent factors for node metastases with $p$ value $\leq 0.05$. Conclusion: Younger age, parity $\geq 4$, stage IIA $>4 \mathrm{~cm}$, diameter of lesion, histopathology adenosquamous, and lymph vascular invasion (+) were risk factors for node metastases and can be used as predictors. (Med J Indones 2004; 13: 113-8)
\end{abstract}

Keywords: cervical cancer, radical hysterectomy, node metastases, case control study, predictor

The presence of lymph node metastasis is considered as the most important prognostic factor for cervical cancer stage IB and IIA. ${ }^{1,2}$ Of 100 stage IB patients, 15 are likely to be node-positive and $45 \%$ of these patients would be cured. The majority of patients is

\footnotetext{
* Department of Obstetrics and Gynecology, Faculty of Medicine, University of Indonesia, Jakarta, Indonesia

${ }^{\dagger}$ Department of Pathological Anatomy, Faculty of Medicine, University of Indonesia, Jakarta, Indonesia

$\S$ Department of Epidemiology, Faculty of Public Health, University of Indonesia, Depok, Indonesia
}

node-negative (85\%), and of these $90 \%$ is cured. ${ }^{3}$ Five year disease free interval decrease significantly from 95\% with node-negative patients to $76 \%$ with nodepositive $(\mathrm{p}<0.002)$. Therefore, the presence of nodalpositivity defines the high-risk group and nodalnegativity the low-risk group.

Detection node metastasis with CT-scan, MRI have high false negative especially for nodes seizing less than $2 \mathrm{~cm},{ }^{4}$ in fact $80 \%$ node metastases were less than $10 \mathrm{~cm}^{5}$

Although many authors have reported risk factors to node metastasis, this study try to identify risk factors 
to node metastasis and then make a formula that can predict probability of node metastasis.

\section{METHODS}

This study was conducted at Dr. Cipto Mangunkusumo Hospital Jakarta from May 1996 until December 2001.There were 183 patients with cervical cancer stage IB and IIA who fulfill inclusion criteria underwent radical hysterectomy and lymphadenectomy. Of those only 158 cases could be evaluated due to incompleteness of surgical procedure, lost of specimens, down stage, paraffin block damage. Stages were determined by physical, gynecological examination, chest x-rays, IVP, cystoscopy, rectoscopy. Ultrasonography, CT-scan or MRI was optional. Peripheral blood test, blood chemistry and ECG was done prior surgery. The surgery was hysterectomy with or without removing one or both ovary and lymphadenectomy. All the specimens were processed and examined at the Department of Pathological Anatomy University of Indonesia.

Study design was a case-control study, patient with node metastasis as a case and who without node metastasis as a control. All clinical and pathological features were evaluated against node metastasis. A model of multivariate was made after bivariate analysis, and then develops a model to predict probability of node metastasis. Statistical program used was Stata Version 6 up-grade.

\section{RESULTS}

There were 43 patients of 158 with node metastasis. The mean age of the study population was 42.19 years with SD \pm 7.78 years, ranging between $20-63$ years. The education range was $0-18$ years, mean education 8.13 years with $\mathrm{SD} \pm 3.88$ years. The parity range was $0-10$, mean parity 4.02 with $\mathrm{SD} \pm 2.23$ (Table 1 ). The diameter of the lesion ranging $10-70 \mathrm{~mm}$, mean 33.84 $\mathrm{mm}$ with SD $\pm 13.37 \mathrm{~mm}$. Most of the patients were stage IB $\leq 4 \mathrm{~cm}(50.63 \%)$ followed by IB $>4 \mathrm{~cm}$ $(21.52 \%)$ IIA $\leq 4 \mathrm{~cm}$ and IIA $>4 \mathrm{~cm}$ (Table 2 ).

Bivariate analysis identified risk factors to node metastasis age $<39$ years $(\mathrm{p}=0.013)$ (Table 3$)$, diameter of the lesion $>4 \mathrm{~cm}(\mathrm{p}=0.005)$, stage IIA
$>4 \mathrm{~cm}(\mathrm{p}=0.000)($ Table 4$)$, moderate $(\mathrm{p}=0.008)$ and poor differentiation $(\mathrm{p}=0.020)$, vascular invasion $(\mathrm{p}=0.001)$, lymphatic invasion $(\mathrm{p}=0.002)$, lymphvascular invasion $(\mathrm{p}=0.009)$ (Table 5). Multivariate analysis revealed that age $(\mathrm{p}=0.024)$, parity $\geq 4(\mathrm{p}=$ $0.001)$, diameter of lesion $(\mathrm{p}=0.000)$, histological type adenosquamous $(p=0.010)$, are independent factors for risk of node metastasis in cervical cancer (Table 6).

\section{DISCUSSION}

Lymph node metastasis can affect 5-year survival in patients with cervical cancer. FIGO in 1998 reported 5 -year survival was $57.1 \%$ on patients with lymph node metastasis and $88.4 \%$ without lymph node metastasis. $^{6}$ Patients with lymph node metastasis found after radical hysterectomy should have adjuvant radiation, chemotherapy or combination of both. However radiation and surgery as a primary treatment yield the same result as shown by Landoni et $\mathrm{al}^{7}$ in their randomized study. Complication will increase if radiation or chemo radiation administered post radical surgery. ${ }^{8,9}$ If lymph node metastasis can be predicted prior surgery the treatment can be well planned and the complication can be avoided. The patient can choose that is suite for her condition. Currently morphological pathology used to predict lymph node metastasis. ${ }^{10}$ Sevin et al in univariate analysis found deep of invasion, diameter of lesion, lymph-vascular infiltration, volume of the tumor were significant predictor lymph node metastasis. Benedetti-Panici et $\mathrm{al}^{11}$ revealed pelvic lymph node metastasis $21 \%$ the lesion $<4 \mathrm{~cm}$ and $23 \%$ if the lesion $>4 \mathrm{~cm}$.

Our study in multivariate analysis revealed younger age, parity $\geq 4$, diameter of lesion, histopathology adenosquamous, and lymph vascular invasion as independent factors for lymph node metastasis with $\mathrm{p}$ value $\leq 0.05$. Based on this multivariate model and equation we can predict the probability lymph node metastasis (Form 1).

Form 1 showing the way to predict probability lymph node metastasis. Conclusion: Younger age, parity $\geq 4$, stage IIA $>4 \mathrm{~cm}$, diameter of lesion, histopathology adenosquamous, and lymph vascular invasion $(+)$ were risk factors for node metastases and can be used as predictors. 
Table 1. Distribution of patients with cervical cancer stage IB and IIA who underwent radical hysterectomy by dempgraphic profile

\begin{tabular}{|c|c|c|}
\hline \multirow{2}{*}{ Demographic profile } & \multicolumn{2}{|c|}{$\mathrm{N}=158$} \\
\hline & $\mathbf{n}$ & $(\%)$ \\
\hline \multicolumn{3}{|l|}{ Age (year) } \\
\hline $20-29$ & 9 & 5.70 \\
\hline $30-39$ & 49 & 31.01 \\
\hline $40-49$ & 71 & 44.94 \\
\hline $50-59$ & 26 & 16.46 \\
\hline$\geq 60$ & 3 & 1.90 \\
\hline Range & $20-63$ & \\
\hline Standard Deviation (SD) & 42.19 & 7.78 \\
\hline \multicolumn{3}{|l|}{ Education (year) } \\
\hline 0 & 10 & 6.33 \\
\hline $1-6$ & 57 & 36.08 \\
\hline $7-9$ & 38 & 24.05 \\
\hline $10-12$ & 47 & 29.75 \\
\hline $13-15$ & 3 & 1.90 \\
\hline$\geq 16$ & 3 & 11.90 \\
\hline Range & $0-18$ & \\
\hline Standard Deviation (SD) & 8.13 & $(3.88)$ \\
\hline \multicolumn{3}{|l|}{ Parity } \\
\hline 0 & 6 & 3.80 \\
\hline $1-3$ & 67 & 42.41 \\
\hline $4-5$ & 48 & 30.38 \\
\hline$\geq 6$ & 37 & 23.42 \\
\hline Range & $0-10$ & \\
\hline Standard Deviation (SD) & 4.02 & $(2.23)$ \\
\hline
\end{tabular}

Table 2. Distribution of patients with cervical cancer stage IB and IIA who underwent radical hysterectomy by clinical characteristic

\begin{tabular}{crr}
\hline \multirow{2}{*}{ Clinical characteristic } & $\mathbf{n}=158$ \\
\cline { 2 - 3 } Diameter of lesion & & $(\%)$ \\
$10-20 \mathrm{~mm}$ & 35 & 22.15 \\
$21-30 \mathrm{~mm}$ & 56 & 35.44 \\
$31-40 \mathrm{~mm}$ & 35 & 22.15 \\
$41-50 \mathrm{~mm}$ & 19 & 12.03 \\
$51-60 \mathrm{~mm}$ & 9 & 5.70 \\
$\geq 61 \mathrm{~mm}$ & 4 & 2.53 \\
Range & $10--70$ & \\
Standard Deviation $(S D)$ & 33.84 & $(13.37)$ \\
& & \\
Stage & & \\
Stage IB $\leq 4 \mathrm{~cm}$ & 80 & 50.63 \\
Stage IB $>4 \mathrm{~cm}$ & 34 & 21.52 \\
Stage IIA $\leq 4 \mathrm{~cm}$ & 34 & 21.52 \\
Stage IIA $>4 \mathrm{~cm}$ & 10 & 6.33 \\
\hline
\end{tabular}

Table 3. Odds ratio (OR) node metastasis of patients with cervical cancer stage IB and IIA by demographic profile (bivariate analysis)

\begin{tabular}{|c|c|c|c|c|c|c|c|}
\hline \multirow{3}{*}{ Demographic profile } & \multicolumn{4}{|c|}{ Metastasis } & \multirow{3}{*}{ OR } & \multirow{3}{*}{$(95 \%$ CI ) } & \multirow{3}{*}{ P Value } \\
\hline & \multicolumn{2}{|c|}{ Negative } & \multicolumn{2}{|c|}{ Positive } & & & \\
\hline & $\mathbf{n}$ & $\%$ & $\mathbf{n}$ & $\%$ & & & \\
\hline \multicolumn{8}{|l|}{ Age(year) } \\
\hline$<39$ & 32 & 27.83 & 21 & 48.84 & 1 & & \\
\hline$\geq 39$ & 83 & 27.17 & 22 & 51.11 & 0.40 & $(0.19 ; 0.84)$ & 0.013 \\
\hline Test for trend (Chi2, $P$ Value) & 6.16 & 0.013 & & & & & \\
\hline \multicolumn{8}{|l|}{ Education (year) } \\
\hline$\leq 9$ & 42 & 36.52 & 11 & 25.58 & 1 & & \\
\hline$>9$ & 73 & 63.48 & 32 & 74.42 & 1.67 & $(0.76 ; 3.69)$ & 0.196 \\
\hline Test for trend (Chi2, $P$ Value) & 1.67 & 0.196 & & & & & \\
\hline \multicolumn{8}{|l|}{ Parity } \\
\hline$\leq 4$ & 76 & 66.09 & 23 & 53.49 & 1 & & \\
\hline$>4$ & 39 & 33.91 & 20 & 46.51 & 1.69 & $(0.82 ; 3.48)$ & 0.146 \\
\hline Test for trend (Chi2, $P$ Value) & 2.11 & 0.146 & & & & & \\
\hline
\end{tabular}


Table 4. Odds ratio (OR) node metastasis of patients with cervical cancer stage IB and IIA by clinical characteristic (bivariate analysis)

\begin{tabular}{|c|c|c|c|c|c|c|c|}
\hline \multirow{3}{*}{ Clinical characteristic } & \multicolumn{4}{|c|}{ Metastasis } & \multirow{3}{*}{ OR } & \multirow{3}{*}{$(95 \%$ CI $)$} & \multirow{3}{*}{$P$ value } \\
\hline & \multicolumn{2}{|c|}{ Negative } & \multicolumn{2}{|c|}{ Positive } & & & \\
\hline & n & $\%$ & $\mathbf{n}$ & $\%$ & & & \\
\hline \multicolumn{8}{|l|}{ Diameter of lesion } \\
\hline$\leq 40 \mathrm{~mm}$ & 98 & 85.22 & 28 & 65.12 & 1 & & \\
\hline$>40 \mathrm{~mm}$ & 17 & 14.78 & 15 & 34.88 & 3.09 & $(1.34 ; 7.12)$ & 0.005 \\
\hline Test for trend (Chi2, $P$ Value) & 7.78 & 0.005 & & & & & \\
\hline \multicolumn{8}{|l|}{ Stage } \\
\hline Stage $\mathrm{Ib} \leq 4 \mathrm{~cm}$ & 64 & 55.64 & 16 & 37.21 & 1 & & \\
\hline Stage $\mathrm{Ib}>4 \mathrm{~cm}$ & 22 & 19.33 & 12 & 27.91 & 2.18 & $(0.88 ; 5.41)$ & 0.840 \\
\hline Stage IIa $\leq 4 \mathrm{~cm}$ & 27 & 23.48 & 7 & 16.28 & 1.04 & $(0.38 ; 2.82)$ & 0.943 \\
\hline Stage IIa $>4 \mathrm{~cm}$ & 2 & 1.74 & 8 & 18.60 & 16.0 & $(2.57 ; 99.45)$ & 0.000 \\
\hline Test for trend (Chi2, $P$ Value) & 6.64 & 0.010 & & & & & \\
\hline
\end{tabular}

Table 5 Odds ratio (OR) node metastasis of patients with cervical cancer stage IB and IIA by pathological characteristic

\begin{tabular}{|c|c|c|c|c|c|c|c|}
\hline \multirow{3}{*}{ Pathological characteristic } & \multicolumn{4}{|c|}{ Metastasis } & \multirow{3}{*}{ OR } & \multirow{3}{*}{$(95 \% \mathrm{CI})$} & \multirow{3}{*}{$P$ value } \\
\hline & \multicolumn{2}{|c|}{ Negative } & \multicolumn{2}{|c|}{ Positive } & & & \\
\hline & $\mathbf{n}$ & $\%$ & $\mathbf{n}$ & $\%$ & & & \\
\hline \multicolumn{8}{|l|}{ Histopathology } \\
\hline Squamous & 75 & 65.22 & 27 & 62.79 & 1 & & \\
\hline Adenocarcinoma & 29 & 25.22 & 6 & 16.95 & 0.57 & $(0.21 ; 1.55)$ & 0,267 \\
\hline Adenosquamous & 10 & 8.70 & 8 & 18.60 & 2.22 & $(0.78 ; 6.31)$ & 0,124 \\
\hline Others & 11 & 0.87 & 2 & 4.65 & 5.56 & $(0.47 ; 66.35$ & 0,127 \\
\hline Test for trend (Chi2, P Value) & 2.08 & 0.149 & & & & & \\
\hline \multicolumn{8}{|l|}{ Differentiation } \\
\hline Good & 30 & 26.09 & 3 & 6.98 & 1 & & \\
\hline Moderate & 36 & 31.30 & 19 & 44.19 & 5.28 & $(1.33 ; 20.87)$ & 0.008 \\
\hline Poor & 49 & 42.61 & 21 & 48.84 & 4.29 & $(1.13 ; 16.28)$ & $\mathbf{0 . 0 2 0}$ \\
\hline Test for trend (Chi2, P Value) & 3.34 & 0.067 & & & & & \\
\hline \multicolumn{8}{|l|}{ Lymphocyte infiltration } \\
\hline Dense & 9 & 7.83 & 6 & 13.95 & 1 & & \\
\hline Moderate & 38 & 33.04 & 15 & 34.88 & 0.59 & $(0.18 ; 1.98)$ & 0.390 \\
\hline Mild & 68 & 59.13 & 22 & 51.16 & 0.49 & $(0.15 ; 1.54)$ & 0.209 \\
\hline Test for trend (Chi2, $P$ Value) & 1.41 & 0.236 & & & & & \\
\hline \multicolumn{8}{|l|}{ Necrosis } \\
\hline Mild & 44 & 38.26 & 17 & 39.53 & 1 & & \\
\hline Moderate & 44 & 38.26 & 12 & 27.91 & 0.71 & $(0.30 ; 1.66)$ & 0.422 \\
\hline Severe & 27 & 23.48 & 14 & 32.56 & 1.34 & $(0.57 ; 3.17)$ & 0.501 \\
\hline Test for trend (Chi2, $P$ Value) & 0.30 & 0.583 & & & & & \\
\hline \multicolumn{8}{|l|}{ Vascular invasion } \\
\hline Negative & 65 & 56.52 & 12 & 27.91 & 1 & & \\
\hline Positive & 50 & 43.48 & 31 & 72.09 & 3.36 & $(1.52 ; 7.40)$ & 0.001 \\
\hline Test for trend (Chi2, $P$ Value) & 10.19 & 0.001 & & & & & \\
\hline \multicolumn{8}{|l|}{ Lymphatic invasion } \\
\hline Negative & 64 & 55.65 & 12 & 27.91 & 1 & & \\
\hline Positive & 51 & 44.35 & 31 & 72.09 & 3.24 & $(1.47 ; 7.13)$ & 0.002 \\
\hline Test for trend (Chi2, $P$ Value) & 9.59 & 0.002 & & & & & \\
\hline \multicolumn{8}{|l|}{ Lymph vascular invasion } \\
\hline Negative & 50 & 43.48 & 9 & 20.93 & 1 & & \\
\hline Positive & 65 & 56.52 & 34 & 79.07 & 2.91 & $(1.25 ; 6.75)$ & 0.009 \\
\hline Test for trend (Chi2, P Value) & 6.76 & 0.009 & & & & & \\
\hline
\end{tabular}


Table 6 Multivariate analysis Odds ratio (OR) node metastasis of patients with cervical cancer stage IB and IIA

\begin{tabular}{lcccccc}
\hline \multicolumn{1}{c}{ Variable } & Coef. & OR & \multicolumn{2}{c}{ 95\% CI } & P value \\
\hline Lymph vascular (positive) & 1.125 & 3.08 & 1.16 & $;$ & 8.18 & 0.024 \\
Diameter of lesion ( mm) & 0.080 & 1.08 & $1.04 ;$ & $; .13$ & 0.000 \\
Age (year) & -0.087 & 0.92 & $0.86 ;$ & 0.98 & 0.012 \\
Parity ( $\geq 4)$ & 1.851 & 6.37 & $2.10 ;$ & $; 9.26$ & 0.001 \\
Adenocarcinoma & 0.315 & 1.37 & $0.43 ;$ & 4.37 & 0.594 \\
Adenosquamous & 1.726 & 5.62 & $1.50 ;$ & $; 20.99$ & 0.010 \\
Others & 2.625 & 13.80 & $0.80 ;$ & $; 6.78$ & 0.070 \\
Constanta & -2.124 & & & & & \\
\hline
\end{tabular}

Form 1 Probability node metastasis in patient with cervical cancer stage IB and IIA

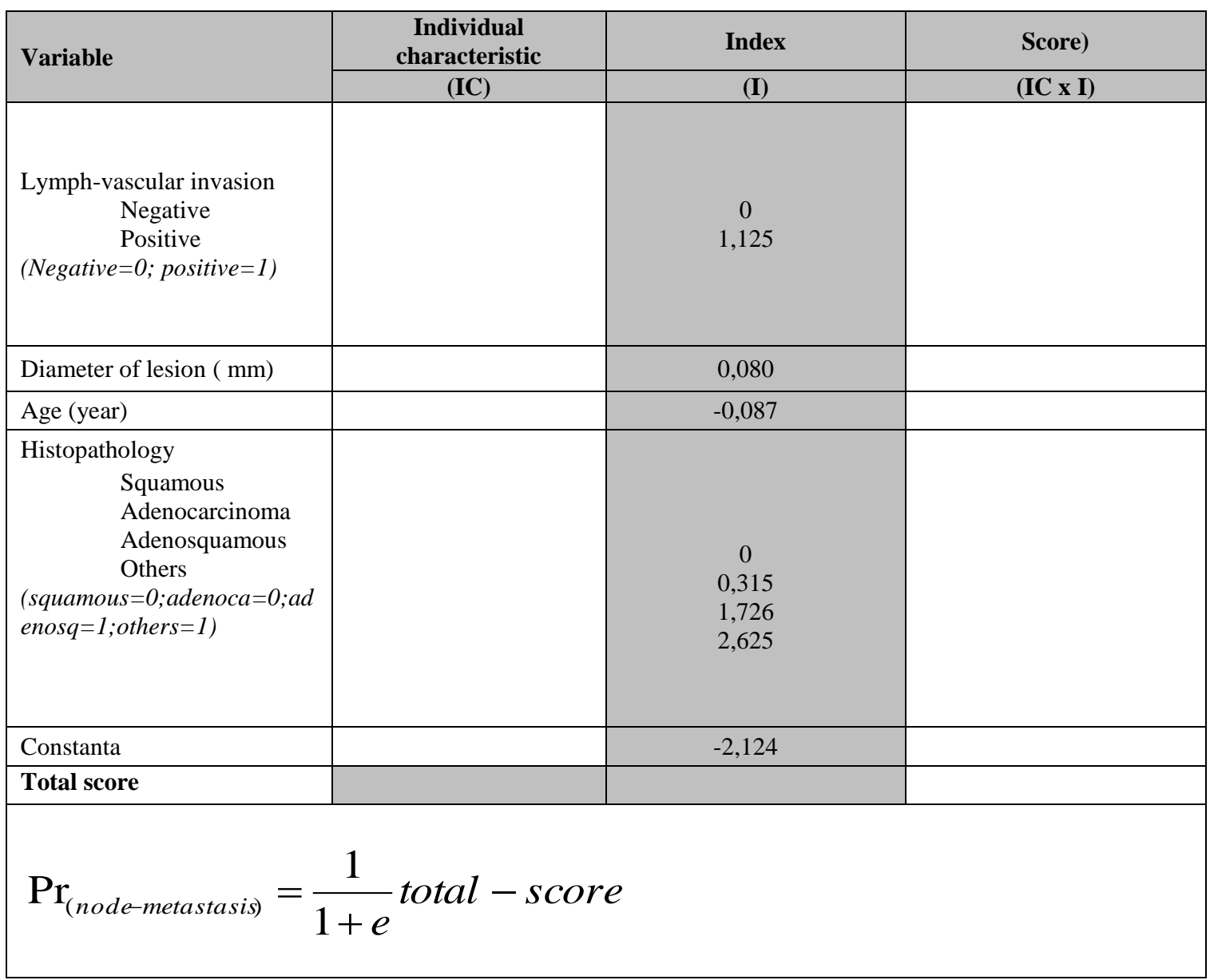

\section{CONCLUSION}

On bivariate analysis of patients with cervical cancer stage IB and IIA who underwent radical hysterectomy, age $<39$ years, diameter of the lesion $>4 \mathrm{~cm}$, stage II $>4 \mathrm{~cm}$, histopathology moderate and poor differentiation, blood and lymphatic vessel invasion were independent variables for lymph node metastasis with $\mathrm{p}$ value $\leq$ 0.05 .

However, on multivariate analysis younger age, parity $\geq 4$, diameter of lesion, histopathology adenosquamous, 
and lymph vascular invasion $(+)$ as independent factors for node metastases with $p$ value $\leq 0.05$. Model to predict the probability of lymph node metastasis can be made.

\section{Acknowledgement}

The authors would like to thank and highly appreciate to the trainees in gynecological oncology and staff of secretariat Division of Oncology Department of Obstetrics and Gynecology, University of Indonesia who have given devotion and care to the patients.

\section{REFERENCES}

1. Kamura T, Tsukamoto N, Tsunechi N, Saito T, Matsuyama T, Akazawa, Nakano H. Multivariate analysis of the histopathologic prognostic factors of cervical cancer in patients undergoing radical hysterectomy. Cancer, 1992, 69: 181-6

2. Sartori E, Fallo L, La Face B, Bianchi UA, Pecorelli S. Extended radical hysterectomy in early-stage carcinoma of the uterine cervix : failaring the radiology. Int $\mathbf{J}$ Gynecol Cancer 1995, 5: 143-7.

3. Thomas GM, Dembo AJ. is there a role for adjuvant pelvic radiation therapy after radical hysterectomy in early stage cervical cancer? Int J Gynecol Oncol 1991, 1: 1-8

4. Kristensen GB, Kaern J, Abeler VM, Hagmar B, Trope CG, Pettersen EO. No prognostic impact of flowcytometric mesured DNA ploidy and s-phase fraction in cancer of the uterine cervix: a prospective study of 465 patients. Gynecol Oncol, 1995,57:79-85.

5. Benedetti-Panici P, Maneschi F, Scambia G, Greggi S, Cutillo G, D'Andrea G, Rabitti C, Coronetta F, Capelli A, dan Mancuso S. Lymphatic spread of cervical cancer: an anatomical and pathological study based on 225 radical hysterectomies with systematic pelvic and aortic lynphadenectomy. Gynecol Oncol 1996, 62:19-24.

6. FIGO Annual report, 1998

7. Landoni F, Maneo A, Colombo A, Placa F, Milani R, Perego P, Favini G, Ferri L, Mangioni C. Randomized study of radical surgery versus radiotherapy for stage IBIIA cervical cancer. Lancet 1997, 350, 535-40.

8. Zola P, Maggino T, Sacco M, Rumore A, Sinistrero G, Maggi R, Landoni F, Foglia G, Sartori E, De Toffoli J, Franchi M, Romagnolo C, Sismondi P. Prospective multicentre study on urologic complications after radicalsurgery with or without radiotherapy in the treatment of stage IB-IIA cervical cancer. Int J Gynecol Cancer 2000; 10: 59-66.

9. Choy D, Wong LC, Sham J, Ngan HYS, Ma HK. Dosetumor response for carcinoma of cervix: an anlysis of 594 patients treated by radiotherapy. Gynecol Oncol 1993, 49: 311-7

10. Sevin BU, Nadji M, Lampe B, Lu Y, Hilsenbeck S, Koechli OR, Averrete HE. Prognostic factors of early stage cervical cancer treated by radical hysterectomy. Cancer 1995, 76:1978-86.

11. Benedetti-Panici P, Maneschi F, Scambia G, Greggi s< Cutillo G, D'Andrea G, Rabitti C, Coronetta F, Capelli A, dan Mancuso S. Lymphatic spread of cervical cancer: an anatomical and pathological study based on 225 radical hysterectomies with systematic pelvic and aortic lynphadenectomy. Gynecol Oncol 1996, 62:19-24. 